\title{
The Assessment of Operational Command And Decision Based on the AHP-Fuzzy Comprehensive Evaluation
}

\author{
Zhao Xue ${ }^{1, a}$, Xiaoming Du, ${ }^{1, b}$ and Guoxu Pei ${ }^{1, c}$ \\ ${ }^{1}$ Ordnance Engineering College, HeBei 050003, China; \\ a623966332@qq.com, bbdar@163.com, c41809817@qq.com
}

Keywords: Operational command and decision, Capability assessment, AHP-FCE.

\begin{abstract}
The assessment of the assessment of operational command and decision, which uses the AHP-FCE to analyze model and judge the single index professionally by building multilevel system of the index evaluation. At the same time, the recursion algorithm was adopted to give the relative degree between object level and evaluation. According to the principle of maximal subordination degree, we can get the merits rating. And adding marking system for the judgment set that the system includes various affecting factors about decision-making. Then, there is a convenient method for integrated assessment of operational command and decision.
\end{abstract}

\section{Introduction}

Operational command under the condition of information, the constantly changing of battle-field situation and a large of information put forward higher requirements for the decision ability of commander and echelon. The command decision-making ability, which is the core of operational command, has a direct impact on the operational command. Therefore, the research to the assessment of operational command decision-making ability, which can provide the basis for the advance of command decision-making ability, has the practical significance. Because of there are many subjective factors, which are fuzziness, and it is quite difficult to do comprehensive and systematic quantitative analysis and comprehensive evaluation. The application of AHP-FCE can greatly solve the problem.

\section{The evaluation index system of operational command and decision}

Before executing programs, we need to build a index system about evaluation. The index system can be built by analyzing the capability requirement and basic function about the system. Command decision-making is the core of operational command, its executing programs is complex that includes the quality of personal, decision rules and the using of the decision mode, and so on. Evaluation index system can be built by the evaluation factors of the operational command and decision, as shown in Figure 1. 


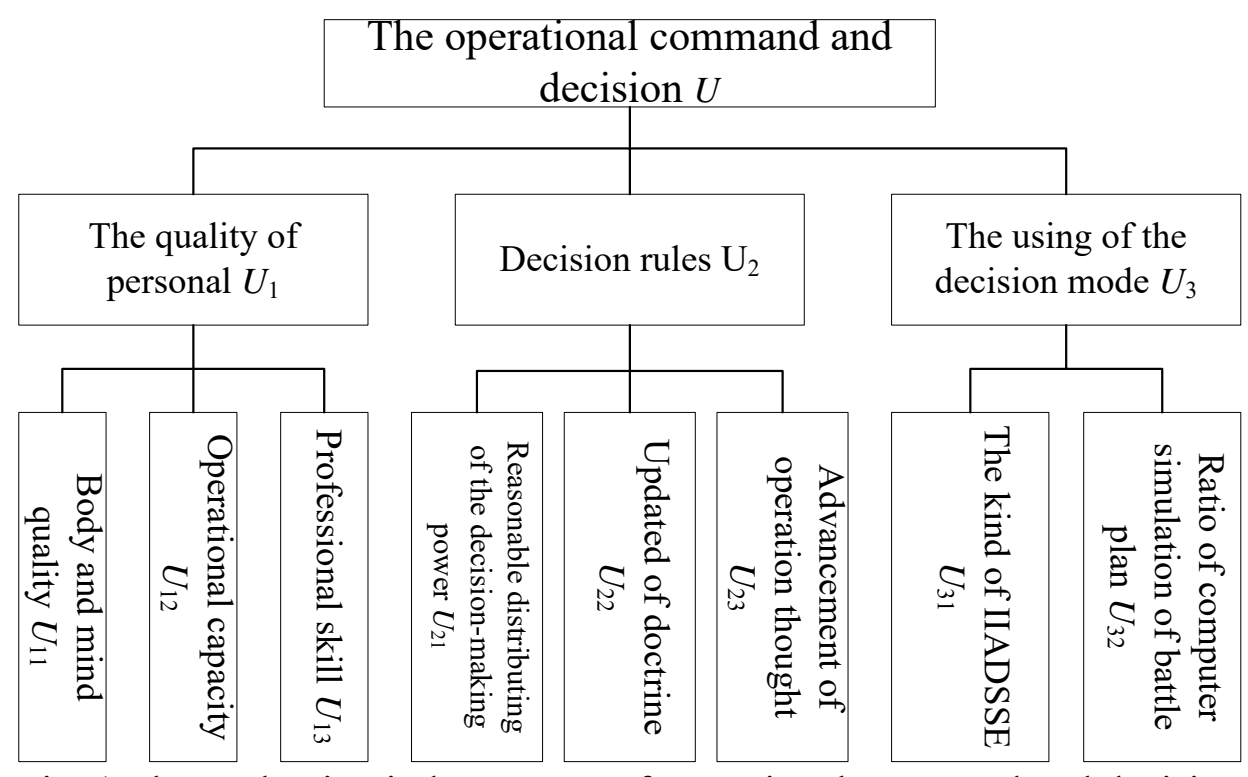

Fig. 1 The evaluation index system of operational command and decision

\section{The evaluation method of operational command and decision}

The key is to build the reasonable evaluation index system and useful evaluation method(model). We found many indexes that are fuzziness by analyzing the index system. So, we evaluate the operational command and decision by using the AHP-FCE.

Determine the index set of evaluation objects. The index set is determined by influence factor $U=\left\{U_{1}, U_{2}, \ldots, U_{n}\right\}$. There are many factors for every primary targets. We mark those secondary indexes as $U_{i}=\left\{U_{i 1}, U_{i 2}, \ldots, U_{i m}\right\}, i \in(1, n)$. According to the fact, we can determine the structure of the multi-factor system.

Index weight. Every index has different effect for the upon level, so we need stipulate a quantitative criterion for the effect, that is called weight. We build the judgment matrix by expert scoring method and pair wise comparison. Using the AHP, we can stipulate the index weight $(\omega)$ for the upon level.

Judgment sets. Judgment sets means the basic standard that assessment panel, evaluates every index by using it can make quantitative analysis in practice. Grade judgment is divided into \{very well, better, general, worse, bad $\}$, and marks it as $V=\left\{v_{1}, v_{2}, \ldots, v_{5}\right\}$. We can get parameter values of $V$ by 10 -scoring method or 100 -scoring method.

Determine the evaluation matrix of each index. Evaluation group firstly judge the most next level indicator by single factor, which means that the mavens vote for single index. Through the result, the judging matrix of single factor is constructed: $R_{k i}=1 / a c_{i j}(i(1, Z), Z$ is the gross of all of the index, $k$ is the $k$-th unit which participate in performance evaluation, $a$ is the number of the mavens, $c_{i j}$ is the number of abstentions which means that the $i-$ th index is evaluated as $v_{j}$. Similarly, the judging matrix of single factor of other index can be calculated.

Determine the comprehensive evaluation model and calculate the comprehensive evaluation results. Above the matrix $R_{k i}$ obtained, and the synthesis operation of fuzzy matrix, the comprehensive evaluation model $B_{k i}=\omega_{\mathrm{i}} \circ R_{k i}$ can be calculated( thereinto: $B_{k i}$ normalization). Because of many factors which have influence on the target, the method of upward recurrence is adopted to calculate the results of every judgment one by one. Then, fuzzy comprehensive evaluation is calculated at last when the results reach to the target layer. The level of target layer can be ascertained by maximum membership principle. Through making the best of thr information of the comprehensive evaluation, the parameter of judgment set is processed as the weight of the comprehensive evaluation results, and specific score of the comprehensive evaluation is calculated, 
which is $D_{k}=\sum_{i=1}^{5} B_{k} \cdot V_{i}$. It can not only distinctly express the comprehensive evaluation result of target layer, but also can compare different evaluation object.

\section{Calculating example}

By AHP-FCE and the evaluation index system of operational command and decision, we compare the command decision-making ability of combat command $A$ of air defense force.

The index weight of effecting command decision-making. The index weight can be determined by using AHP and asking for the professor. For example, the first level includes three indexes that includes the quality of personal, the decision rules and the using of the decision model. The following is matrix of pairwise comparison by expert decision: $\left[\begin{array}{ccc}1 & 2 & 3 \\ 1 / 2 & 1 & 2 \\ 1 / 3 & 1 / 2 & 1\end{array}\right]$, the largest eigenvalue $\lambda \max =3.009$, random conformance rate $\mathrm{CR}=\mathrm{C} / \mathrm{R}=0.008<0.1$, so the matrix is reasonable, the weight $\omega \mathrm{A}=(0.539,0.2973,0.1637)$, so the next levels $\omega 1=(0.5571,0.1226,0.3203)$, $\omega 2=(0.648,0.1222,0.2298), \omega 3=(0.6198,0.3802)$.

Evaluation matrix. In this example, the judgment set $V=\{$ very well, better, general, worse, bad $\}$, The parameter $V=\{9,7,5,3,1\}$ is determined by 10 -scoring method. We invite ten professors to judge eight indexes of second level. The results are shown in Table 1.

Table 1 Results

\begin{tabular}{cccccc}
\hline Air defense combat command structure $A$ & Very well & Better & General & Worse & Bad \\
\hline Body and mind quality $U_{11}$ & 0 & 3 & 6 & 1 & 0 \\
Operational capacity $U_{12}$ & 1 & 4 & 5 & 0 & 0 \\
$\quad$ Professional skill $U_{13}$ & 2 & 5 & 1 & 1 & 1 \\
$\begin{array}{c}\text { Reasonable distributing of the } \\
\text { decision-making power } U_{21}\end{array}$ & 1 & 2 & 5 & 2 & 0 \\
$\quad \begin{array}{c}\text { Updated of doctrine } U_{22} \\
\text { Advancement of operation thought }\end{array}$ & 1 & 3 & 4 & 1 & 1 \\
$\quad \begin{array}{l}U_{23} \\
\text { The kind of IIADSSE } U_{31}\end{array}$ & 0 & 1 & 5 & 2 & 2 \\
Ratio of computer simulation of battle plan & 1 & 3 & 5 & 1 & 1 \\
$U_{32}$ & & 6 & 0 & 0 \\
\hline
\end{tabular}

The following is the judgment matrix:

$$
\begin{aligned}
R_{1} & =\left[\begin{array}{ccccc}
0 & 0.3 & 0.6 & 0.1 & 0 \\
0.1 & 0.4 & 0.5 & 0 & 0 \\
0.2 & 0.5 & 0.1 & 0.1 & 0.1
\end{array}\right] \\
R_{2} & =\left[\begin{array}{ccccc}
0.1 & 0.2 & 0.5 & 0.2 & 0 \\
0.1 & 0.3 & 0.4 & 0.1 & 0.1 \\
0 & 0.1 & 0.5 & 0.2 & 0.2
\end{array}\right] \\
R_{3} & =\left[\begin{array}{ccccc}
0 & 0.3 & 0.5 & 0.1 & 0.1 \\
0.1 & 0.3 & 0.6 & 0 & 0
\end{array}\right]
\end{aligned}
$$

Appraisal Calculation. According to the above conclusion, $B i=\omega_{i} \circ R_{i}$ ( $\circ$ is a compositional operation, it means $M(*,+)$ operator). We can make the compositional operation for the $A$ :

$B_{1}{ }^{\prime}=\omega_{1} \circ R_{1}=(0.0763,0.3763,0.4276,0.0877,0.032)$ 


$$
\begin{aligned}
& B_{2}^{\prime}=\omega_{2} \circ R_{2}=\left(\begin{array}{llll}
0.077, & 0.1892, & 0.4878,0.1878, & 0.0582
\end{array}\right) \\
& B_{3}{ }^{\prime}=\omega_{3} \circ R_{2}=\left(\begin{array}{llll}
0.038 & 0.3,0.538,0.062,0.062
\end{array}\right)
\end{aligned}
$$

So, we can know the judgement matrix of primary level is

$$
R_{A}=\left[\begin{array}{ccccc}
0.0763 & 0.3763 & 0.4277 & 0.0877 & 0.032 \\
0.077 & 0.1892 & 0.4878 & 0.1878 & 0.0582 \\
0.038 & 0.3 & 0.538 & 0.062 & 0.062
\end{array}\right]
$$

So,

$$
B_{A}=\omega_{\mathrm{A}} \circ R_{A}=\left(\begin{array}{llll}
0.0702, & 0.3082, & 0.4636, & 0.1132,0.0448
\end{array}\right)
$$

According to the parameter of judgment set, the final grade of performance evaluation is

$$
D_{A}=B_{A} \cdot V^{T}=6.1093
$$

Firstly, analyzing the vector quantity $B$ of fuzzy evaluation, we can get the meaning that command and decision-making efficiency of $A$ is general. In a similar way, the grades of quality of personal, decision rules and the using of the decision model are 6.3697, 5.6604, 6.068. According to the grade, we can see that the person have high quality. But the decision-making is unreasonable, so the grade of the command decision-making efficiency is lower. If we improved the command decision-making efficiency, we should make the distributing of the decision-making power be reasonable and operation though be advance.

\section{Conclusion}

This paper uses the AHP-FCE to analyze the command decision-making ability, which is based on the assessment of operational command and decision. Through the command system and evaluation method quantificational echelon and describe the command decision-making ability. It can provide the basis for the construction of operational command and decision and command system. Through the analysis of examples, the model of fuzzy analytic hierarchy process meet the needs of actual combat, and can conquer the uncertainty factors of system. Which have good feasibility, usability and generality, and have some reference function to the assessment of other problems..

\section{References}

[1] Wang Renfang, Wang Juhai, Lv Qiang, et. Evaluation of Air Defense Forces Group Command Organization Personnel Quality Based on Fuzzy Comprehensive Judgment [J]. Ship Electronic Engineering, 2011,31(6) .

[2] Cheng Qiyue. Operation command decision making and strategy analysis[M] .Bei Jing: Military Science Press,2004.

[3] Xie Jijian, Liu Chengping. The method and application of fuzzy mathematics[M].Wu Han: Huazhong University of Science and Technology Press,2005.

[4] Liu Jun, Jia Honghui. Application of FAHP in effectiveness evaluation of air defense missile weapon system[J]. Computer and Engineering and Design,2007(16) .

[5] Ding Yongli, Li Yingqi, Tian Ye. Fuzzy AHP evaluation model of missile weapon system effectiveness eavaluation[J]. SiChuan Ordnance Journal,2010(6) . 